\title{
A differential-outcomes effect using hedonically nondifferential outcomes with delayed matching to sample by pigeons
}

\author{
Holly C. Miller, Andrea M. Friedrich, Randi J. Narkavic, and Thomas R. Zentall \\ University of Kentucky, Lexington, Kentucky
}

\begin{abstract}
When differential outcomes follow correct responses to each of two comparison stimuli in matching to sample, relative to the appropriate control condition, higher matching accuracy is typically found, especially when there is a delay between the sample and the comparison stimuli. In two experiments, we examined whether this differential-outcomes effect depends on using outcomes that differ in hedonic value (e.g., food vs. water). In Experiment 1 , we found facilitated retention when a blue houselight followed correct responses to one comparison stimulus and a white houselight followed correct responses to the other, prior to nondifferential presentations of food. In Experiment 2, we found facilitated retention again when a blue houselight followed correct responses to one comparison stimulus and a tone followed correct responses to the other, prior to nondifferential presentations of food. The results of both experiments indicate that the differential-outcomes effect does not depend on a difference in hedonic value of the differential outcomes, and they suggest that outcome anticipations consisting of relatively arbitrary but differential stimulus representations can serve as cues for comparison choice.
\end{abstract}

In a conditional discrimination, an initial or sample stimulus indicates which test or comparison stimulus is correct. When different outcomes follow correct responding to each of the comparison stimuli, faster acquisition of the conditional discrimination is sometimes found (Trapold, 1970). Furthermore, relative to an appropriate control condition involving nondifferential outcomes, insertion of a delay between the offset of the sample and the onset of the comparison stimuli with differential outcomes typically results in improved delayed matching performance (Peterson, 1984).

The use of differential outcomes has important practical implications for the acquisition and retention of new associations (Maki, Overmier, Delos, \& Gutmann, 1995). It also has important theoretical implications, because the outcome, which appears to serve as a cue for comparison choice, comes after the choice. Thus, it appears that the anticipation of the outcome can serve as an effective choice cue, but the nature of that anticipation is not well understood.

In most demonstrations of the differential-outcomes effect, the outcomes have differed in quantitative or qualitative value-for example, one versus five pellets of food (Carlson \& Wielkiewicz, 1976), 1.0 versus .5 probability of a food outcome (Kruse \& Overmier, 1982), food versus water (Honig, Matheson, \& Dodd, 1984), or wheat versus corn (Edwards, Jagielo, Zentall, \& Hogan, 1982). If so, the differential value of the outcomes may produce differential behavior to the samples, which may serve as an additional cue for comparison choice (Alling, Nickel, \& Poling, 1991; Brodigan \& Peterson, 1976; Peterson \& Trapold, 1980).

For example, Zentall, Sherburne, and Steirn (1992) trained pigeons with a differential-outcomes procedure involving differential probabilities of reinforcement (1.0 and 0 ) with correction trials. Zentall et al. found that when a delay was inserted between the sample and the comparison choice, there was a strong differential-outcomes effect (relative to the appropriate control group). However, they also found that the pigeons in the differential-outcomes group pecked at a high rate to the sample that was associated with a high rate of reinforcement and virtually not at all to the sample that was associated with the absence of reinforcement. To determine whether differential response rates to the samples were responsible for the differential-outcomes effect, Zentall et al. trained pigeons to respond to samples nondifferentially by including trials on which pecks to the sample associated with no-food outcomes were immediately followed by reinforcement (without comparison presentation). For these pigeons, peck rates on trials that ended in food were similar to those that ended in no food, yet they performed better than pigeons that were nondifferentially reinforced, and at a level of accuracy that was similar to the level displayed by pigeons that were differentially reinforced and that exhibited differential responding to the samples. Thus, although hedonically different outcomes can result in

T. R. Zentall, zentall@uky.edu 
differential sample responding, differential response rates cannot account for the differential-outcomes effect.

A second mechanism that may mediate the differentialoutcomes effect is the conditioned appetitive value of the sample stimulus that signals an anticipated differentially preferred outcome. It is well established that outcome expectation can affect behavior (Crespi, 1942; Tinklepaugh, 1928); thus, if two samples elicit differential incentive motivation (Hull, 1952), differential motivation could serve as the basis for comparison choice.

Control of comparison choice by differential outcome value was demonstrated by Astley, Peissig, and Wasserman (2001). In one of their experiments, each of two samples was associated with a different number of food pellets (one and five) and each of two other samples was associated with different delays to reinforcement ( 1 and $15 \mathrm{sec}$ ). On test trials, they found generalization of comparison choice between the number of pellets and the delay (i.e., there was transfer from, e.g., the larger number of pellets to the shorter delay). But as the authors acknowledged, relative peck rates were confounded with motivational state (the pigeons pecked more to samples that signaled either five pellets or a 1-sec delay). Thus, it is not clear whether it was the value of the stimulus or the pigeons' differential keypecking that mediated comparison choice.

In a more direct test of differential motivation associated with the two conditional cues, Fedorchak and Bolles (1986) trained rats with differential outcomes that had presumed nondifferential hedonic value. They trained rats to press the right lever in the presence of one cue - a tone or a clickerand the left lever in the presence of the other cue for the same water reinforcement. For their differential-outcomes group, the houselight flashed for $0.5 \mathrm{sec}$ following correct responses to one of the levers but not to the other. For the nondifferential-outcomes group, the houselight flashed for $0.5 \mathrm{sec}$ following half of the correct responses to either lever. Fedorchak and Bolles found that the differentialoutcomes group acquired the conditional discrimination significantly faster than did the nondifferential-outcomes group (see also Friedman \& Carlson, 1973).

In a similar experiment involving matching to sample with pigeons, Kelly and Grant (2001) also trained with differential outcomes that had presumed nondifferential hedonic value. They followed correct comparison choice following one sample with one distinctive color on the response keys and correct comparison choice following the other sample with a different distinctive color and found faster acquisition by the differential-outcomes group than by a nondifferential-outcomes control group. Although rate of acquisition provides a useful measure of group differences, its transitory nature makes it susceptible to individual differences in rates of learning and position and stimulus biases. For this reason, rate of acquisition generally has been found to be a less reliable measure of the differential-outcomes effect than has matching accuracy tested with varying delays between the sample and comparison stimuli (see, e.g., Sherburne \& Zentall, 1995; Zentall \& Sherburne, 1994). Furthermore, it is possible that when assessed during acquisition, differential outcomes have an indirect effect on the rate of learning by increasing the discriminability of the sample or comparison stimuli.

Delay testing not only provides a steady-state measure of the effect of the differential outcomes, but also allows additional time within a trial for the anticipation of the trial outcome to emerge. Moreover, because the measure is taken only after acquisition, after the samples and comparisons have been well discriminated, the observed effects of differential outcomes are more likely to be directly attributable to the anticipation of the outcome, rather than to sample or comparison discriminability.

In another attempt to assess the effect of differential outcomes that were presumed to have nondifferential hedonic values, D. A. Williams, Butler, and Overmier (1990) used differential feeder location as the differential outcome. With their procedure, a key on the far left served as the sample, and two keys in the middle, one above the other, served as comparison stimuli. There were two feeders mounted on the right, one adjacent to each of the comparison keys. For the differential-outcomes group, following presentation of one sample, a response to the top comparison key was reinforced with food presented at the top feeder. Following presentation of the other sample, a response to the bottom comparison key was reinforced with food presented at the bottom feeder. For the nondifferential-outcomes group, choice of the correct comparison at either location was reinforced with food at either feeder, randomly selected.

Although D. A. Williams et al. (1990) did not report acquisition rates, when delays were introduced, they found higher matching accuracy for the differential-outcomes group than for the nondifferential-outcomes group. However, anticipation of a spatially distinctive outcome could have promoted anticipatory movement toward the appropriate feeder. With the more typical matching procedure in which the correct comparison for a particular sample could appear in either of two locations, the location of the correct comparison stimulus cannot be anticipated. Furthermore, we have found that when feeder location is used as a differential outcome, pigeons show strong (idiosyncratic) feeder preferences (Friedrich \& Zentall, 2004). Thus, it is likely that spatially distinct but otherwise identical feeders may not provide outcomes with nondifferential hedonic values.

\section{EXPERIMENT 1}

The purpose of Experiment 1 was to examine the effect of a variable delay (between the offset of the sample and the onset of the comparison stimuli) for a group of pigeons trained with hedonically nondifferential differential outcomes using a nonspatial comparison discrimination (location of the correct comparison varied randomly) and to determine whether such differential outcomes can facilitate memory relative to a group trained with nondifferential outcomes. To provide a salient differential-outcome cue following correct comparison choices, we used houselights of different colors (white and blue) that appeared immediately following choice of the correct comparison and $1 \mathrm{sec}$ prior to the delivery of food (common for all correct responses and at a common location). 


\begin{abstract}
Method
Subjects

The subjects were 8 White Carneaux pigeons (Columba livia), 6-14 years of age, of undetermined sex, from the Palmetto Pigeon Plant (Sumter, SC). All of the pigeons had previous experience with simple simultaneous discriminations. The pigeons were housed in individual cages ( $35 \mathrm{~cm}$ wide, $35 \mathrm{~cm}$ high, and $76 \mathrm{~cm}$ deep) and were maintained on a 12:12-h light:dark cycle. The birds had free access to water and grit and were maintained at $85 \%$ of their free-feeding weight for the duration of the experiment. The animals were cared for in accordance with University of Kentucky animal care guidelines.
\end{abstract}

\section{Apparatus}

The experiment was conducted in a test chamber (BRS/LVE, Laurel, MD) $31 \mathrm{~cm}$ high, $35 \mathrm{~cm}$ deep (across the response panel), and $33 \mathrm{~cm}$ wide, measured from the response panel to the back wall of the chamber. The response panel contained three horizontally aligned, circular response keys, $2.5 \mathrm{~cm}$ in diameter and spaced $8.0 \mathrm{~cm}$ apart center to center. Each key was illuminated by a projector (Industrial Electronic Engineers, Model 10, Van Nuys, CA) that was mounted behind the panel. Red, green, yellow, and blue hues were projected onto the response keys through Kodak Wratten filters, nos. 26, 60, 9, and 38, respectively. The test chamber contained two houselights: one that emitted white light and was located on the response panel $1.0 \mathrm{~cm}$ above the center response key, another that emitted blue light and was located in the center of the ceiling. Reinforcement - 1.5 -sec access to Purina Pro Grain - was provided through a $5.0 \times 5.0 \mathrm{~cm}$ aperture situated $1.0 \mathrm{~cm}$ directly beneath the center response key and $0.5 \mathrm{~cm}$ above the floor of the chamber. An externally mounted exhaust fan provided air circulation and masked extraneous sounds.

\section{Procedure}

Zero-delay conditional discrimination training. The 8 pigeons were randomly assigned to two groups, 4 birds each: the differential-outcomes (DO) group and the nondifferential-outcomes (NDO) group. The pigeons were trained on an identity matching-tosample task. For 2 birds from each group, the training sample and comparison stimuli were red and green. For the remaining pigeons, the stimuli were yellow and blue.

Each trial began with the presentation of one of the colored sample stimuli on the center response key. After the pigeon pecked the sample key 10 times, the sample was turned off, and comparison stimuli appeared on the left and right side keys. Choice of the matching comparison stimulus resulted in the illumination of one of the two houselights for $1.0 \mathrm{sec}$ prior to 1.5 -sec access to the mixed grain. A 10 -sec intertrial interval followed, during which the houselight was turned off and all three of the response keys were illuminated with white light. Choice of the incorrect comparison resulted in only the 10 -sec intertrial interval.

For the DO pigeons, the color of the houselight that followed each correct comparison choice was correlated with the color of the correct comparison. For 2 of the DO pigeons, the blue houselight always followed the correct choice of the red (or yellow) comparisons, and the white houselight always followed the correct choice of the green (or blue) comparisons. For the other 2 DO pigeons, the blue houselight always followed correct choice of the green (or blue) comparisons, and the white houselight always followed the correct choice of the red (or yellow) comparisons. For the NDO pigeons, one of the two houselights was randomly lit following each correct response to either pair of comparison stimuli. Training sessions (96 trials each) continued until the pigeons attained a $90 \%$ level of accuracy or better for two consecutive sessions.

Delay testing. The pigeons were tested for matching-to-sample accuracy following a comparison onset delay of $0,2,4$, or $8 \mathrm{sec}$. Each session consisted of 96 trials, with the four delays represented equally and with presentation order randomly determined. During the delays, the test chamber remained dark. In all other respects, testing was

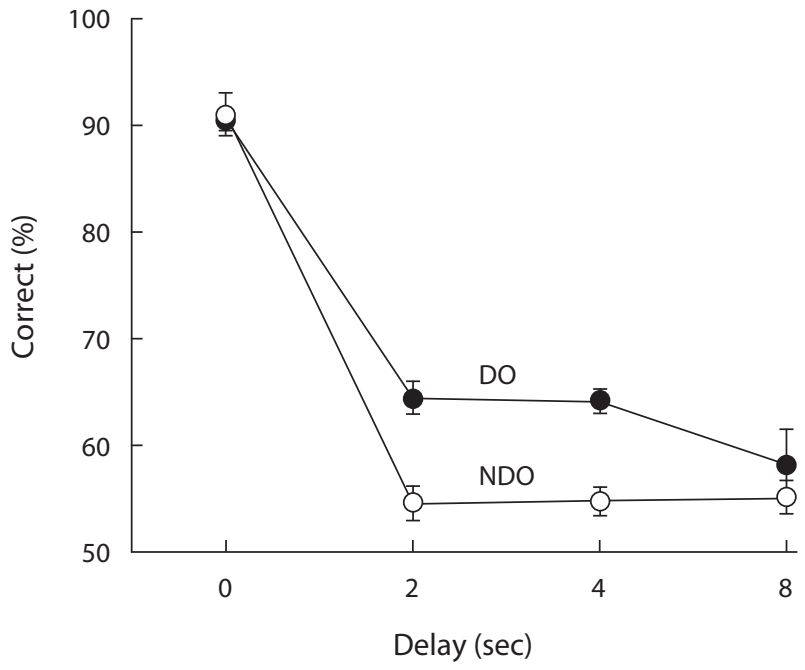

Figure 1. Experiment 1. Retention functions for the differentialoutcomes (DO) group and the nondifferential-outcomes (NDO) group. Error bars indicate the standard errors of the means.

the same as training. Each pigeon completed 12 test sessions. In all analyses, the .05 level of statistical significance was adopted.

\section{Results}

\section{Acquisition}

One pigeon in the DO group became sick during training and was dropped from the study. The remaining 7 pigeons attained criterion with a range of 5-23 sessions (mean $=10.6$ sessions). Pigeons in the DO group reached criterion in 8.4 sessions, whereas those in the NDO group reached criterion in 12.2 sessions. A $t$ test performed on the acquisition scores for pigeons in the two groups indicated that the difference was not statistically significant $[t(5)=1.25, p=.24]$.

\section{Delay Testing}

Matching accuracy was pooled over the 12 test sessions and is presented in Figure 1 for each group at each delay interval. As can be seen in Figure 1, matching accuracy by pigeons in the DO group was higher than it was in the NDO group at delays of 2 and $4 \mathrm{sec}$ but not at 0 and $8 \mathrm{sec}$. A mixedfactors two-way ANOVA was performed on the pooled data from the test sessions with delay $(0,2,4$, and $8 \mathrm{sec})$ and group (DO and NDO) as factors. The analysis indicated that there was a significant effect of group $[F(1,5)=10.71, p=$ $.02]$ and a significant effect of delay $[F(3,15)=62.95, p<$ $.001]$; however, the group $\times$ delay interaction did not reach statistical significance $[F(3,15)=1.54, p>.05]$.

\section{Discussion}

The purpose of this experiment was to determine whether we could obtain a differential-outcomes effect with outcomes that do not have differential hedonic value. When differently colored houselights that followed correct comparison choices were correlated with the color of the sample and correct comparison stimulus (the DO group), 
significantly more accurate delay performance was found than when the differently colored houselights were uncorrelated with the color of the sample and correct comparison stimulus (the NDO group). This experiment differed from the earlier differential-outcomes experiment (D. A. Williams et al., 1990) involving spatial delayed matching, in that the pigeons could not mediate the delay by approaching the correct comparison location during the delay. Although the difference in performance between the DO group and the NDO group was reliable, the magnitude of the effect was not particularly large. In fact, mean matching accuracy for the DO group at 2-, 4-, and 8-sec delays never exceeded $65 \%$ correct. It may be that the stimulus difference between the blue and white houselights was not sufficient to produce a large differential-outcomes effect.

\section{EXPERIMENT 2}

The purpose of Experiment 2 was to further test the hypothesis that a differential-outcomes effect can be obtained with differential outcomes that do not differ in hedonic value. To produce a differential-outcomes effect greater than that found in Experiment 1, outcomes were used in Experiment 2 that were more different from each other than were the blue and white houselights used in Experiment 1. In Experiment 2, a 1-kHz tone was substituted for the white houselight used in Experiment 1. It was assumed that the difference in modality between the blue houselight and the tone would make them more different from each other than the within-dimension difference between the blue and white houselights.

\section{Method}

Subjects

The subjects were 8 White Carneaux pigeons similar in all respects to those used in Experiment 1. Their housing and care were also similar to those aspects of Experiment 1.

\section{Apparatus}

The apparatus used in Experiment 2 was similar to the apparatus in Experiment 1, with the following exceptions: The three response keys on the response panel were square $(2.5 \times 2.5 \mathrm{~cm})$ and were separated by $0.8 \mathrm{~cm}$.

The 1.0-kHz tone produced by a Hewlett Packard 209A oscillator was amplified by an MPA-31 P.A. 20-W amplifier and presented through a $4-\Omega$ speaker located behind the response panel. The tone was used as one of the differential outcomes for correct comparison choice. The blue houselight used in Experiment 1 served as the other differential outcome.

\section{Procedure}

Zero-delay conditional discrimination training. For all subjects, a training trial began with the presentation of either a red or green sample light on the center key. Ten pecks to this key darkened it and resulted in the immediate presentation of green and red on the side keys. The location of comparison choices was counterbalanced over trials. A single peck to the matching comparison stimulus resulted in presentation of the tone or the blue houselight for $3 \mathrm{sec}$. Reinforcement $(1.5 \mathrm{sec})$ was presented $1.5 \mathrm{sec}$ after the onset of the tone or light (a bit longer than the $1.0 \mathrm{sec}$ used in Experiment 1). All trials were followed by a 10 -sec intertrial interval, during which the white houselight was lit.

Four of the pigeons were randomly assigned to the DO group and the remaining pigeons were assigned to the NDO group. For pigeons in the DO group, the tone or blue houselight was presented differentially following the correct comparison choice (depending on the sample color). For half of the pigeons, the tone followed a correct choice of the red comparison and the blue houselight followed a correct choice of green. For the remaining pigeons, these stimulus presentations were reversed. For pigeons in the NDO group, the tone or blue houselight was presented nondifferentially (i.e., with equal probability) following correct comparison choices of both red and green comparisons. Each training session consisted of 96 trials, with an equal number of red and green sample trials. Training sessions were conducted once a day, 6 days a week. The pigeons were trained to a criterion of $85 \%$ correct or better on each of the sample types for three consecutive sessions.

Delay testing. When criterion was reached, a delay was interpolated between the offset of the sample and the onset of the comparison stimuli. Testing sessions were similar to the training sessions, with the exception that the duration of the retention interval varied from trial to trial $(0,2,4$, or $8 \mathrm{sec})$. There was an equal number of trials at each retention interval, and the different retention intervals were randomly presented during each testing session. There were 24 testing sessions, conducted with 0-, 2-, 4-, or 8-sec delays.

\section{Results}

\section{Acquisition}

The DO group acquired the matching task (10.0 sessions) somewhat faster than did the NDO group (14.2 sessions), but the difference was not statistically reliable $[F(1,6)=5.59, p=.06]$.

\section{Delay Testing}

Matching accuracy was pooled over the 24 test sessions, and the data for each group at each delay are presented in Figure 2. As can be seen from Figure 2, matching accuracy by pigeons in the DO group was higher than it was in the NDO group at the 2-, 4-, and 8-sec delays. A two-way mixed-factors ANOVA performed on the data with delay and group as factors indicated a significant main effect of group $[F(1,6)=12.76, p=.01]$, a significant main effect of delay $[F(3,18)=49.45, p<.0001]$, and a significant group $\times$ delay interaction $[F(3,18)=$ $4.18, p=.02]$.

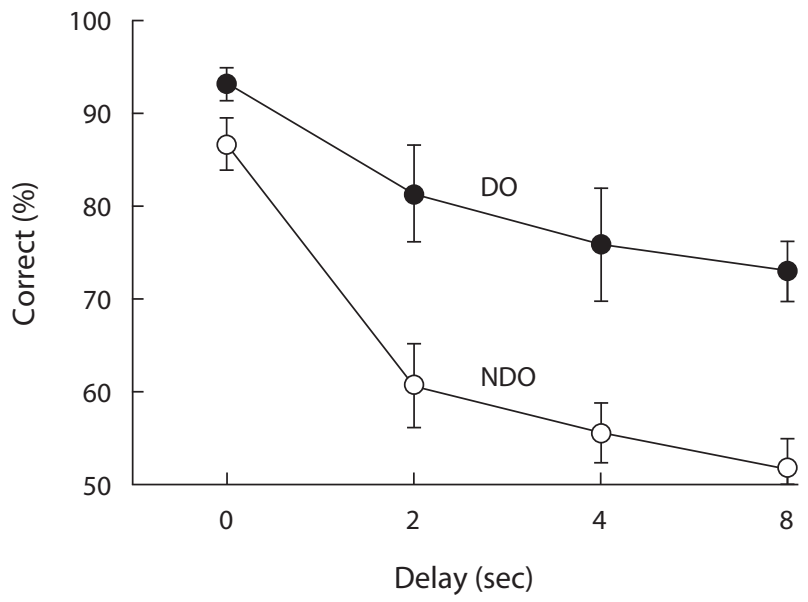

Figure 2. Experiment 2. Retention functions for the differentialoutcomes (DO) group and the nondifferential-outcomes (NDO) group. Error bars indicate the standard errors of the means. 


\section{Discussion}

The results of Experiment 2 confirm and extend the results of Experiment 1. Pigeons that had differential outcomes involving hedonically nondifferential tone and houselight stimuli showed better matching accuracy when delays were introduced. Presenting the two different outcomes in two different modalities - one visual (a houselight), the other auditory (a tone) - appeared to make the anticipated outcomes somewhat more memorable. Although the retention functions for the NDO groups in Experiments 1 and 2 look quite similar, the DO group in Experiment 2 showed better delayed matching accuracy than did the DO group in Experiment 1. This difference may account for the fact that there was a significant group $X$ delay interaction in Experiment 2 but not in Experiment 1. In Experiment 2, the group difference continued to grow with increasing delay, whereas, in Experiment 1, the relatively poorer performance by the DO group resulted in a performance floor and may have prevented a significant interaction from emerging.

\section{GENERAL DISCUSSION}

The results of both experiments extend the results of earlier research with hedonically nondifferential differential outcomes, in which better retention was found for pigeons trained with differential outcomes than for pigeons trained with nondifferential outcomes (D. A. Williams et al., 1990). However, unlike in the D. A. Williams et al. study, in the present experiments, the location of the correct comparison stimulus could not be anticipated when the sample was presented.

The rationale for the present experiments was to test the hypothesis that the differential-outcomes effect may depend on the use of outcomes likely to differ in hedonic value (e.g., food vs. water or high- vs. low-probability food presentation). If the differential-outcomes effect does depend on outcomes with differential hedonic value, differential incentive motivation may result and provide discriminative stimuli capable of mediating the delay and of guiding choice behavior. The fact that a differentialoutcomes effect can be found in delayed matching with arbitrary light and tone outcomes suggests that outcomes of differential hedonic value are not necessary to produce a differential-outcomes effect. These results, together with the results of earlier research (Kelly \& Grant, 2001; D. A. Williams et al., 1990) and of transfer tests-in which samples associated with differential outcomes in one conditional discrimination can substitute for samples associated with the same differential outcomes in a different conditional discrimination (e.g., Edwards et al., 1982) - further suggest that the expectation of an outcome can serve - at least in part - as the basis for choice in much the same way as a concurrent or recently presented cue, such as a sample.

The results of the present experiments further illustrate that acquisition rate is not a particularly reliable dependent measure of the effects of differential outcomes with the matching-to-sample procedure. The results suggest that delays presented between the sample offset and comparison onset are more sensitive to a differentialoutcomes effect. Although the reason for this difference between acquisition and delay testing is not clear, it may be that individual differences among pigeons in acquisition of matching to sample - as well as preexperimental comparison-stimulus and side preferences - may mask the detection (by statistical analysis) of the effects of differential outcomes during acquisition.

One may notice a similarity between the procedure used in the present experiments and that used in experiments on marking (e.g., Lieberman, Davidson, \& Thomas, 1985). In the marking procedure, if a distinctive stimulus is presented following a response at a point prior to the outcome (reinforcement or its absence) associated with that response, it often facilitates the acquisition of a simple discrimination, relative to the absence of the distinctive stimulus. Similarly, in the experiments on nonhedonically differential outcomes, a distinctive stimulus appears between the correct response and reinforcement.

However, important differences between these two procedures suggest that the two effects result from different underlying mechanisms. First, the differential-outcomes procedure involves a conditional discrimination in which the different responses conditional on the sample are followed by different events, whereas the marking procedure involves a simple simultaneous discrimination in which the single event that follows the response is not differential. Second, the critical feature of the marking procedure is that the distinctive event that follows the response occurs regardless of whether the choice was correct or incorrect (B. A. Williams, 1994), whereas, in the differential-outcomes procedure, the distinctive event follows only correct choices. B. A. Williams proposed that marking helps to bridge the delay between the response and the outcome, whereas, in most cases, the differentialoutcomes effect does not involve such a delay.

Interestingly, when Lieberman et al. (1985, Experiment 3, marked-same group) marked correct but not incorrect responses - a procedure not unlike the differentialoutcomes procedure - it also facilitated acquisition. However, with this kind of marking, the mark functioned as a conditioned reinforcer, distinguishing correct from incorrect responses, whereas, in the case of differential outcomes, the outcomes distinguish one kind of correct response from the other.

The importance of the present findings (as well as those of Kelly \& Grant, 2001, and D. A. Williams et al., 1990) concerns the nature of the cues provided by anticipation of the differential outcomes. In the traditional differentialoutcomes experiment, it is possible that the association of samples with different hedonic outcomes can produce differential hedonic states, and those different states may be the added source of discriminative stimuli at the time of choice (a form of momentary state-dependent learning). The present findings indicate that the expectation of arbitrary stimuli, such as differently colored houselights (Experiment 1) or a houselight versus an auditory stimulus (Experiment 2) can also serve as anticipatory discriminative stimuli at the time of choice. That the expectation of arbitrary stimuli (that do not differ in hedonic value) can 
serve as discriminative stimuli suggests that the processes involved in the differential-outcomes effect do not necessarily rely on differential motivational states and thus have a component that is more cognitive in nature.

\section{AUTHOR NOTE}

This research was supported by National Institute of Mental Health Grant 63726. Correspondence concerning this article should be addressed to T. R. Zentall, Department of Psychology, University of Kentucky, Lexington, KY 40506-0044 (e-mail: zentall@uky.edu).

\section{REFERENCES}

Alling, K., Nickel, M., \& Poling, A. (1991). The effects of differential and nondifferential outcomes on response rates and accuracy under a delayed-matching-to-sample procedure. Psychological Record, 41, 537-549.

Astley, S. L., Peissig, J. J., \& Wasserman, E. A. (2001). Superordinate categorization via learned stimulus equivalence: Quantity of reinforcement, hedonic value, and the nature of the mediator. Journal of Experimental Psychology: Animal Behavior Processes, 27, 252-268.

Brodigan, D. L., \& Peterson, G. B. (1976). Two-choice conditional discrimination performance of pigeons as a function of reward expectancy, prechoice delay, and domesticity. Animal Learning \& Behavior, 4, 121-124.

Carlson, J. G., \& Wielkiewicz, R. M. (1976). Mediators of the effects of magnitude of reinforcement. Learning \& Motivation, 7, 184-196.

CRESPI, L. P. (1942). Quantitative variation of incentive and performance in the white rat. American Journal of Psychology, 55, 467-517.

EDWARds, C. A., Jagielo, J. A., Zentall, T. R., \& Hogan, D. E. (1982). Acquired equivalence and distinctiveness in matching to sample by pigeons: Mediation by reinforcer-specific expectancies. Journal of Experimental Psychology: Animal Behavior Processes, 8, 244-259.

FedorchaK, P. M., \& Bolles, R. C. (1986). Differential outcome effect using a biologically neutral outcome difference. Journal of Experimental Psychology: Animal Behavior Processes, 12, 125-130.

Friedman, G. J., \& CARLson, J. G. (1973). Effects of a stimulus correlated with positive reinforcement upon discrimination learning. Journal of Experimental Psychology, 97, 281-286.

Friedrich, A. M., \& Zentall, T. R. (2004). Pigeons shift their preference toward locations of food that take more effort to obtain. Behavioural Processes, 67, 405-415.

Honig, W. K., Matheson, W. R., \& Dodd, P. W. D. (1984). Outcome expectancies as mediators for discriminative responding. Canadian Journal of Psychology, 38, 196-217.

Hull, C. L. (1952). A behavior system: An introduction to behavior theory concerning the individual organism. New Haven, CT: Yale University Press.

KeLly, R., \& GRANT, D. S. (2001). A differential outcomes effect using biologically neutral outcomes in delayed matching-to-sample with pigeons. Quarterly Journal of Experimental Psychology, 54B, 69-79.

Kruse, J. M., \& Overmier, J. B. (1982). Anticipation of reward omission as a cue for choice behavior. Learning \& Motivation, 13, 505-525.

Lieberman, D. A., Davidson, F. H., \& Thomas, G. V. (1985). Marking in pigeons: The role of memory in delayed reinforcement. Journal of Experimental Psychology: Animal Behavior Processes, 11, 611-624.

Maki, P., Overmier, J. B., Delos, S., \& Gutmann, A. J. (1995). Expectancies as factors influencing conditional discrimination performance of children. Psychological Record, 45, 45-71.

Peterson, G. B. (1984). How expectancies guide behavior. In H. L. Roitblat, T. G. Bever, \& H. S. Terrace (Eds.), Animal cognition (pp. 135-148). Hillsdale, NJ: Erlbaum.

Peterson, G. B., \& Trapold, M. A. (1980). Effects of altering outcome expectancies on pigeons' delayed conditional discrimination performance. Learning \& Motivation, 11, 267-288.

Sherburne, L. M., \& Zentall, T. R. (1995). Pigeons transfer between conditional discriminations with differential outcomes in the absence of differential-sample-responding cues. Animal Learning \& Behavior, 23, 273-279.

TinkLePaUGH, O. L. (1928). An experimental study of representative factors in monkeys. Journal of Comparative Psychology, 8, 197-236.

Trapold, M. A. (1970). Are expectancies based upon different positive reinforcing events discriminably different? Learning \& Motivation, 1, 129-140.

Williams, B. A. (1994). Conditioned reinforcement: Neglected or outmoded explanatory construct? Psychonomic Bulletin \& Review, 1, 457-475.

Williams, D. A., Butler, M. M., \& Overmier, J. B. (1990). Expectancies of reinforcer location and quality as cues for a conditional discrimination in pigeons. Journal of Experimental Psychology: Animal Behavior Processes, 16, 3-13.

Zentall, T. R., \& Sherburne, L. M. (1994). Role of differential sample responding in the differential outcomes effect involving delayed matching by pigeons. Journal of Experimental Psychology: Animal Behavior Processes, 20, 390-401.

Zentall, T. R., Sherburne, L. M., \& Steirn, J. N. (1992). Development of excitatory backward associations during the establishment of forward associations in a delayed conditional discrimination by pigeons. Animal Learning \& Behavior, 20, 199-206.

(Manuscript received April 29, 2008; revision accepted for publication September 16, 2008.) 